\title{
Respuesta psicofisiológica en un salto táctico paracaidista HAHO. Caso de Estudio
}

\author{
Clemente-Suárez VJ.1,2,3, Delgado-Moreno R. .,3, Gonzalez-Gómez B. ${ }^{2,3}$, Robles-Pérez JJ.,4
}

Sanid. mil. 2015; 71 (3): 179-182; ISSN: 1887-8571

\begin{abstract}
RESUMEN
Esta investigación tiene por objeto analizar la respuesta psicofisiológica de un paracaidista en un salto HAHO. Se analizó a un saltador experimentado de la Brigada Paracaidista del Ejército de Tierra Español en un salto HAHO, salto a 18000 pies y apertura a 16000 pies. Antes y después del salto se evaluaron parámetros de fuerza muscular, activación cortical, creatinfosfokinasa y glucosa, percepción subjetiva de esfuerzo y habilidades motrices finas específicas. Durante el salto se evaluó la frecuencia cardiaca, la modulación autonómica y la acelerometría. Los datos muestran como un salto HAHO provocó un aumento de la modulación simpática, alcanzando valores de frecuencia cardiaca máxima de 175 pulsaciones, una disminución de la activación cortical y de la fuerza muscular, un aumento de la creatinfosfokinasa, un mantenimiento de la glucosa, una percepción subjetiva de esfuerzo inferior a la respuesta orgánica evaluada y ningún efecto sobre la habilidad motriz fina especifica del combatiente.
\end{abstract}

PALABRAS CLAVE: HAHO, salto paracaidista, creatinfosfokinasa, frecuencia cardiaca, lactato, combatiente.

\section{Psychophysiological response in a HAHO tactical parachute jump. A case study}

SUMMARY: This research aimed to study the psychophysiological response in a HAHO parachute jump. A HAHO jump, jump at 18,000 feet and opening at 16,000 feet, was evaluated in one warfighter of the Paratrooper Brigade of the Spanish Army. Before and after the HAHO jump parameters of muscle strength, cortical arousal, blood levels of creatinekinase and glucose, rated of perceived exertion and specific fine motor skills were assessed. During the jump heart rate, autonomic modulation and accelerometry were evaluated. The data shows as a HAHO jump caused an increased sympathetic modulation, reaching values of maximum heart rate of 175 beats per minute, a decreased in cortical arousal and muscle strength, an increased blood levels of creatinekinase, a maintenance of blood glucose, a rated of perceived exertion lower than the assessed organic response and no effect on specific fine motor skills of the warfighter.

KEYWORDS: HAHO, Parachute jump, Creatinekinase, Heart rate, Lactate, Warfighter.

\section{INTRODUCCIÓN}

El combate es una de las situaciones más estresantes a las que el organismo del ser humano puede enfrentarse, ya que el combatiente tiene que hacer frente a un gran número de situaciones que pueden poner en peligro tanto su integridad física como su vida. Recientes estudios han mostrado la activación de mecanismos innatos de defensa como el sistema de lucha-huida en situaciones de combate simétrico y asimétrico ${ }^{1-3}$. Este hecho provoca en los combatientes una activación del sistema nervioso simpático que aumenta la producción de energía por vías metabólicas anaeróbicas y un aumento de la frecuencia cardiaca para proveer rápidamente de energía a la musculatura ${ }^{2,4}$. Sin embargo, esta alta activación orgánica no es percibida por el combatiente ya que reporta valores bajos de percepción subjetiva de esfuerzo (RPE) en este tipo de combates ${ }^{1,3}$. También ha sido evalua-

${ }^{1}$ Universidad Europea de Madrid. Facultad de Ciencias de la Actividad Física y el Deporte. Departamento de Motricidad, Rendimiento Humano y Gestión del Deporte. Madrid. España.

2 Club Triatlón Tritoledo. Toledo. España.

${ }^{3}$ Centro de Estudios Superiores de Combate Aplicado. Toledo. España.

${ }^{4}$ Cte. Cuerpo General de la Armas, Especialidad Fundamental de Infantería. Cuartel General de Fuerzas Ligeras. Ejército de Tierra. Madrid. España.

Dirección para correspondencia: Vicente Javier Clemente Suárez. Departamento de Motricidad, Rendimiento Humano y Gestión del Deporte. Facultad de Ciencias de la Actividad Física y el Deporte. Universidad Europea de Madrid. Calle Tajo, s/n, 28670 Villaviciosa de Odón, Madrid, España e-mail: vctxente@yahoo.es

Recibido: 17 de noviembre de 2014

ptado: 2 de marzo de 2015 do como el estrés de combate produce una disminución en el procesamiento de la información y fatiga del sistema nervioso central, posiblemente debido al alto número de incertidumbres a controlar por el combatiente en el campo de batalla ${ }^{5}$. En estas situaciones, el combatiente puede interpretar esas incertidumbres como posibles elementos desde los cuales puede aparecer una acción hostil que comprometa su integridad, provocando un estado de ansiedad en el cual el cerebro es sobreestimulado ${ }^{6}$, haciendo estas situaciones muy estresantes para el combatiente ${ }^{7}$. Esta respuesta ansiógena afecta tanto a la respuesta psicofisiológica como a la memoria operativa del combatiente ${ }^{7}$.

Dentro de las unidades de élite de los actuales ejércitos encontramos las brigadas paracaidistas, las cuales además de enfrentarse a las mismas situaciones de combate que el resto de la infantería, tienen que realizar la incursión en la zona de operaciones realizando un salto paracaidista. En esta acción el combatiente tiene que saltar con todo el equipo y pertrechos para el combate lo que dificulta aún más la acción táctica. Dentro de los diferentes saltos tácticos que pueden realizar estas unidades encontramos los saltos automáticos, manuales y por último los saltos a gran altitud HALO (High Altitud Low Opening) y HAHO (High Altitud High Opening). En el salto HAHO el combatiente es lanzado desde un avión a alturas superiores a los 18000 pies y la apertura la realiza a alta cota al poco tiempo después de saltar del avión. Debido a la gran altura de salto el paracaidista tiene que ser equipado con máscara de oxígeno y un equipo pesado para aguantar las condiciones ambientales del salto. 
Estudios previos realizados en paracaidistas han mostrado la activación del sistema nervioso simpático en saltadores, independientemente de su experiencia ${ }^{8}$, altos niveles de arousal ${ }^{9}$, aumentos de hormonas estresantes como el cortisol y valores bajos de testosterona ${ }^{10}$, aunque actuales estudios muestras un gran aumento de la reactividad de la testosterona durante saltos a 14000 pies $^{11}$. Otros autores han analizado las principales lesiones acaecidas en saltadores tanto en paracaidismo tanto civil ${ }^{12}$ como militar ${ }^{13,14}$. A pesar de estos estudios el análisis de la respuesta psicofisiológica en saltos de alta cota como el HAHO no ha sido analizado previamente, a pesar de ser uno de los saltos más extremos que se realizan y uno de los más peligrosos. Por ello esta investigación tiene como objetivo el analizar la respuesta cardiaca, metabólica, muscular, cortical, autonómica, ansiógena, la percepción subjetiva de esfuerzo y la coordinación motriz fina específica de un saltador experimentado en un salto táctico HAHO.

\section{MATERIAL Y MÉTODOS}

\section{Sujeto de estudio}

Se analizó a un saltador experimentado (más de 100 saltos) de la Brigada Paracaidista del Ejército de Tierra Español (26 años; $80 \mathrm{~kg} ; 188 \mathrm{~cm}$ ). El combatiente iba equipado con el uniforme, botas, paracaídas manual, paracaídas de reserva, equipo de combate, sistema de navegación y sistema de respiración con un peso de $70 \mathrm{~kg}$. Todas las pruebas realizadas se llevaron a cabo de acuerdo con la declaración de Helsinki, el sujeto fue informado de los test a realizar y dio su consentimiento firmado.

\section{Toma de muestras e instrumentos de evaluación}

El paracaidista realizó un salto HAHO saltando a la altura de 18000 pies y abriendo el paracaídas a 16000 pies. Antes de realizar el equipamiento para el salto y después del reagrupamiento al realizar la toma de tierra del salto HAHO se realizaron las siguientes pruebas con el siguiente orden.

- Percepción subjetiva de esfuerzo (RPE) con la escala 6-2015.

- Saturación de oxígeno en sangre mediante un pulsioxímetro (PO 30 Beurer Medical).

- Concentración sanguínea de lactato tomando una muestra de $5 \mu$ de sangre capilar de un dedo del combatiente y analizado posteriormente con el sistema de lactato Lactate ProArkay, Inc. system (Kyoto, Japan).

- Concentración sanguínea de glucosa tomando una muestra de $5 \mu \mathrm{l}$ de sangre capilar de un dedo del combatiente $\mathrm{y}$ analizado con un analizador portátil (One Touch Basic, LifeScan Inc. Madrid)

- Concentración sanguínea de creatinkinasa (CK) tomando una muestra de $32 \mu \mathrm{l}$ de sangre capilar de un dedo del combatiente. La muestra sanguínea se analizaba inmediatamente mediante el sistema Reflotron Plus. Roche Diagnostics S.L. (Sant Cugat del Vallès, Barcelona).

- Fuerza isométrica de prensión de mano con dinamómetro Takkei.
- Fuerza explosiva de piernas mediante sistema de acelerometría Sensorize (Sanro Electromedicina, Madrid) consistente en la realización de una batería de 6 saltos, 2 Squat Jump (SJ), 2 saltos con contramovimiento (CMJ) y 2 saltos con contramovimiento y ayuda de brazos $(\mathrm{ABK})^{2,3}$.

- Activación cortical y fatiga del Sistema Nervios Central (SNC) mediante el sistema Lafayette Instrument Flicker Fusion Control Unit (Model 12021), mediante el cual se determinó el Umbral de Frecuencia Crítica de Fusión (UFCF) de acuerdo a previos estudios ${ }^{1-4}$. El análisis del UFCF es un método utilizado frecuentemente como medida de la fatiga del SNC, incrementos en ese valor indican un aumento de la activación cortical y el procesamiento de la información, por el contrario, una disminución de los valores mostraría fatiga del SNC y una reducción en la eficiencia en los sistemas de procesamiento de la información ${ }^{16-18}$.

- Habilidad motriz fina específica, mediante el tiempo de municionamiento de 15 balas $9 \mathrm{~mm}$ parabelum en un cargador de pistola Beretta.

Durante la maniobra el combatiente analizado llevó un sistema de GPS (SPI Elite; GPSports Systems, Canberra, Australia) junto con una banda polar T34 sincronizada para evaluar la frecuencia cardiaca. La aceleración del combatiente fue registrada utilizando el acelerómetro triaxial con un rango de muestreo de $100 \mathrm{~Hz}$. El sistema GPS fue activado previo a la fase de equipamiento y se paró después de recoger el equipo al tomar tierra. Posteriormente los datos fueron descargados en un ordenador y analizados mediante el programa Team AMS software (GPSports, V1.2, Canberra, Australia) ${ }^{5}$. Además el combatiente llevaba un pulsómetro polar V800 con función de análisis RR para el análisis de la variabilidad de la frecuencia cardiaca. Posteriormente al salto se analizaron los siguientes parámetros de variabilidad de frecuencia cardiaca mediante el programa $\mathrm{Ku}-$ bios HRV software (University of Kuopio. Kuopio, Finland):

- RMSSD (ms): Es la raíz cuadrada del valor medio de la suma de las diferencias al cuadrado de todos los intervalos R-R sucesivos.

- HF (u. n): Banda de alta frecuencia.

- LF (u. n.): Banda de baja frecuencia.

La variable independiente de este estudio fue el salto paracaidista HAHO, las variables dependientes fueron: RPE, saturación de oxígeno, concentración sanguínea de lactato, glucosa y CK, fuerza isométrica manual, valores de saltos SJ, CMJ y $\mathrm{ABK}$, activación cortical, tiempo de municionamiento de cargador de beretta, frecuencia cardiaca, RMSSD, HF y LF.

\section{RESULTADOS}

Los resultados de frecuencia cardiaca y velocidades obtenidas con el dispositivo GPS se muestran en la tabla 1, divididos en 3 fases, fase de equipamiento previa a la entrada en el avión, fase vuelo en avión hasta la zona de lanzamiento y por último la fase de salto HAHO desde la salida del avión hasta la toma de tierra. 


\section{Respuesta psicofisiológica en un salto táctico paracaidista HAHO. Caso de Estudio}

En la tabla 2 se muestran los resultados del análisis de la variabilidad de la frecuencia cardiaca.

Después del salto los valores de RPE, concentración sanguínea de lactato y $\mathrm{CK}$ y el tiempo de municionamiento aumentaron, sin embargo, los valores de fuerza isométrica, fuerza de piernas, concentración sanguínea de glucosa, activación cortical y saturación de oxígeno disminuyeron (tabla 3 ).

Tabla 1. Resultados de frecuencia cardiaca y velocidad de movimiento del combatiente.

\begin{tabular}{|llcc|}
\hline Fase & & $\begin{array}{c}\text { Frecuencia Cardiaca } \\
\text { (ppm) }\end{array}$ & Velocidad (km/h) \\
\hline \multirow{4}{*}{ Equipamiento } & Mínimo & 89 & 0 \\
& Máximo & 149 & 8.4 \\
& Media & 108 & 0.4 \\
\multirow{4}{*}{ Avión } & Mínimo & 84 & - \\
& Máximo & 156 & - \\
& Media & 104 & - \\
\multirow{3}{*}{ HAHO } & Mínimo & 115 & 8.7 \\
& Máximo & 175 & 366.0 \\
& Media & 144 & 123.3 \\
\hline
\end{tabular}

Tabla 2. Parámetros de variabilidad de la frecuencia cardiaca analizados.

\begin{tabular}{|llll|}
\hline Fase & RMSSD (ms) & HF (u. n.) & LF (u. n.) \\
\hline Equipamiento & 35.3 & 38.0 & 61.0 \\
Avión & 26.4 & 15.9 & 84.0 \\
HAHO & 18.7 & 13.9 & 86.1 \\
\hline
\end{tabular}

RMSSD: Raíz cuadrada del valor medio de la suma de las diferencias al cuadrado de todos los intervalos R-R sucesivos; HF: Banda de alta frecuencia; LF: Banda de baja frecuencia; u. n.: unidades normalizadas.

Tabla 3. Resultados de los parámetros analizados previos y posteriores al salto $\mathrm{HAHO}$.

\begin{tabular}{|lccc|}
\hline Parámetro & Pre & Post & \% Diferencia \\
\hline RPE (-) & 6 & 11 & 83,3 \\
Fuerza isométrica (n) & 48,3 & 47,8 & $-1,0$ \\
SJ (cm) & 39 & 37 & $-5,1$ \\
CMJ (cm) & 45 & 41 & $-8,9$ \\
ABK (cm) & 53 & 52 & $-1,9$ \\
Lactato (mmol/l) & 1,6 & 2,8 & 75,0 \\
Glucosa (mmol/l) & 9,9 & 8 & $-19,2$ \\
CK (UI) & 98 & 259 & 164,3 \\
UFCF (Hz) & 40,1 & 39,6 & $-1,2$ \\
Saturación Oxigeno (\%) & 97 & 96 & $-1,0$ \\
Tiempo municionamiento (s) & 41,13 & 42,31 & 2,9 \\
\hline
\end{tabular}

RPE: Percepción subjetiva de esfuerzo; SJ: Squat Jump; CMJ: Salto con contramovimiento; ABK: Salto con contramovimiento y ayuda de brazos; CK: Creatinfosfokinasa; UFCF: Umbral de Frecuencia Crítica de Fusión.

\section{DISCUSION}

El objetivo del presente estudio era analizar la respuesta psicofisiológica de un saltador experimentado en un salto táctico
HAHO. El análisis de los resultados muestra como este tipo de saltos operativos provocan una alta respuesta tanto fisiológica como psicológica.

Los valores de frecuencia cardiaca analizados muestran como desde la fase de equipamiento el combatiente tiene una alta respuesta cardiovascular con respecto a la velocidad de movimiento de este, hecho debido al gran peso que tiene que movilizar para la colocación y transporte del equipo tanto de combate como de lanzamiento. En la fase de vuelo hasta la zona de lanzamiento se alcanzaron frecuencias cardiacas máximas momentos previos al salto, correspondiente a un $80 \%$ de la frecuencia cardiaca máxima teórica para la edad del combatiente ${ }^{19}$. Este resultado es similar al de previos estudios realizados con saltos civiles y a menor cota que el HAHO, en los cuales se mostró una alta activación simpática previa al salto, independientemente de la experiencia del saltador y un aumento brusco de la frecuencia cardiaca del saltador $^{8}$. En la caída del salto se monitorizó la frecuencia cardiaca máxima de toda la maniobra, correspondiendo al $90 \%$ de la frecuencia cardiaca máxima del combatiente 9 . La frecuencia cardiaca media durante el salto HAHO se situaría dentro de una zona de trabajo aeróbico ligero ${ }^{20}$. La modulación autonómica también se vio modificada dependiendo de la fase analizada, encontrando una mayor modulación simpática en el salto HAHO, después en la fase de vuelo del avión y por último en el equipamiento como muestran los altos valores de las variables RMSSD y $\mathrm{HF}^{21}$. Se puede ver que mientras más se acerca el momento de salto la modulación simpática es mayor, activándose el organismo para el momento del salto.

Después del salto HAHO se pudo observar una ligera disminución del UFCF, lo que muestra una disminución de activación cortical y es considerado como un síntoma de fatiga del sistema nervioso central. Este dato es contradictorio con los obtenidos en saltadores civiles, ya que en ese caso se evaluó un aumento del arousal de los saltadores ${ }^{9}$. Esta diferencia puede reflejar una inclinación individual a realizar procedimientos operativos estandarizados que han sido muy entrenados y requieren menor esfuerzo, esto maximizaría el rendimiento del sujeto minimizando los recursos cognitivos empleados en el proceso ${ }^{22,23}$. El descenso en la activación cortical del saltador puede estar relacionado con la disminución de los valores de fuerza evaluados después del salto, ya que el estímulo que provoca la contracción muscular es iniciado en el cerebro y las alteraciones del sistema nervioso central disminuyen la habilitad para enviar voluntariamente señales a la unión neuromuscular ${ }^{24}$. Además los altos valores de CK evaluados después del salto muestran un aumento de la destrucción muscular, factor que puede alterar la normal contracción y manifestación de fuerza de la musculatura del paracaidista. Estos valores de $\mathrm{CK}$ fueron inferiores a los evaluados en combatientes en situaciones de combate tradicional o simétrico ${ }^{3}$ y los obtenidos por corredores después de realizar una maratón de montaña ${ }^{24}$, lo que muestra un impacto medio a nivel muscular de este tipo de saltos operativos.

El salto HAHO también provocó un ligero aumento de los metabolismos anaeróbicos alcanzando los resultados de lactato sanguíneo evaluados valores inferiores al umbral anaeróbico o el OBLA $^{25}$. Los valores alcanzados en la concentración sanguínea de lactato son similares a los evaluados en situaciones de combate asimétrico y simétrico ${ }^{1-3}$, pero inferiores a los obtenidos en 
combates a corta distancia y cuerpo a cuerpo, en los cuales la activación muscular es mayor y los valores de lactato son mayores ${ }^{4}$. A pesar de la concentración de lactato cercana al OBLA y de la alta frecuencia cardiaca obtenida en el salto, el valor de RPE del combatiente no reflejaba esta respuesta fisiológica, marcando un valor de 13 puntos. Este resultado pone de manifiesto que el saltador no es consciente realmente de la carga fisiológica que provoca el salto HAHO, mostrando una percepción subjetiva de esfuerzo inferior a la respuesta orgánica evaluada. Además los valores de glucosa sanguínea a pesar de registrar una disminución, se mantienen altos después del salto HAHO, estos resultados pueden ser debidos a la activación del sistema de lucha-huida activado por el aumento en la modulación simpática debido a la situación estresante que es el salto. Esta situación provoca un aumento de los metabolismos energéticos anaeróbicos para proveer energía para poder cubrir cualquier posible incidencia que pueda poner en peligro la integridad del sujeto.

Al igual que la concentración glucosa se mantuvo alta, los valores de saturación de oxigeno se mantuvieron cercanos a los valores basales después del salto HAHO, resultado que pudo ser posible gracias a la utilización de sistemas de respiración exógenos durante el salto. Por último, y a pesar del incremento en la respuesta fisiológica, la habilidad motriz fina específica del combatiente no se vio alterada, mostrando valores de tiempo de municionamiento de cargador similares a las encontradas en situación basal. Estos resultados están en contra de las teorías clásicas y de estudios previos realizados en situaciones de estrés ${ }^{26}$, lo que pone de manifiesto una correcta instrucción en situaciones de estrés por parte del paracaidista.

\section{Limitaciones de estudio}

La principal limitación del estudio es el hecho de realizarse un estudio de caso único. Además se podrían haber utilizado mediciones no indirectas de la activación cortical, pero debido a la realización de las tomas de muestras fuera del laboratorio en un salto real la utilización de material para la toma de datos fue muy limitada.

\section{Aplicación práctica}

Los datos de este estudio, siempre teniendo en cuenta la limitación de que es un caso único, marca las bases para una preparación de objetivos fisiológicos, anatómicos y psicológicos del saltador de alta cota, lo cual puede utilizarse para implementar programas de formación específicos para este tipo de intervenciones.

\section{CONCLUSIONES}

El salto HAHO provocó un aumento de la modulación simpática, una disminución de la activación cortical y de la fuerza muscular, un aumento de la concentración sanguínea de creatinfosfokinasa, una mantenimiento de valores de glucosa sanguínea, de saturación de oxígeno, una percepción subjetiva de esfuerzo inferior a la respuesta orgánica evaluada y ningún efecto sobre la habilidad motriz fina especifica del combatiente.

\section{BIBLIOGRAFÍA}

1. Clemente-Suárez V. y Robles-Pérez J. Organic response in a combat simulation. Sanidad Mil. 2012; 68(2). 97-100

2. Clemente-Suárez V. y Robles-Pérez J. Psycho-physiological response of soldiers in urban combat. An Psychol. 2013 29(2): 598-603.

3. Clemente-Suarez V y Robles J. Analysis of physiological markers, cortical activation and manifestations of force in a simulated combat. Arch Med Deporte. 2012; 149: 594-600.

4. Clemente-Suárez V y Robles-Pérez J. Psycho-physiological response in diferent combat situations. Editorial Académica Española. Saarbrücken. Deutschland. 2012.

5. Clemente-Suárez V y Robles-Pérez J. Mechanical, physical and physiological analysis of symmetrical and asymmetrical combat. J Strength Cond Res 2012; 27(9): 2420-6. doi: 10.1519/JSC.0b013e31828055e9.

6. Martens R, Vealey R, Burton D. Competitive anxiety in sport. Champaign: Human kinetics, 1990. 342-5.

7. Taverniers J, Van Ruysseveldt, J, Smeets T Y von Grumbkow J. High-intensity stress elicits robust cortisol increases and impairs working memory and visuo-spatial declarative memory in Special Forces candidates: A field experiment. Stress 2010; 13(4): 324-34.

8. Amber L, Allisona JC, Peresa C, Boettgera U, Leonbachera PD, Hastingsb E. Fight, flight, or fall: Autonomic nervous system reactivity during skydiving. Pers Indiv Differ 2012; 53(3): 218-23.

9. Hetland A, Vittersø J. The feelings of extreme risk: exploring emotional quality and variability in skydiving and BASE jumping. J Sport Behav 2012; 35(2): 154-80.

10. Chatterton R, Vogelsong K, Lu Y, Hudgens G. Hormonal responses to psychological stress in preparing for skydiving. J Clin Endocrinol Metab 1997; 82: 2503-09.

11. Shrestha S. "Study of testosterone reactivity and its variance in response to skydiving" (March 22, 2013). Innovate UNO. Paper 2.

12. Esser SM, Baima, J, Hirschberg R. Falling for Sport: A Case Report of Skydiving and SCI. Curr. Sports Med. Rep: 2013; 12(1): 7-10.

13. Knapik JJ, Steelman R, Grier T, Graham B, Hoedebecke K, Rankin S, et al. Military parachuting injuries, associated events, and injury risk factors. Aviat Space Environ Med 2011; 82:797-804.

14. Guo W, Ghen Y, Yang Y, Qu G, Liu D, Song Q. Analysis of risk factor for military parachuting injuries among Chinese air force cadet pilots. Appl Mech Mat 2013; 423-426: 1778-81.

15. Borg G. Perceived exertion as an indicator of somatic stress. Scan J Rehab Med 1970; 2(2), 92-8.

16. Li Z, Jiao K, Chen M, Wang C. Reducing the effects of driving fatigue with magnitopuncture stimulation. Acc Anal Prev. 2004; 36: 501-5.

17. Costa G. Evaluation of workload in air traffic controllers. Ergonomics. 1993; 6: 1111-20.

18. Saito $\mathrm{S}$. Does fatigue exist in a quantitative measurement of eye movements? Ergonomics. 1992; 35: 607-15.

19. Tanaka, H., Monahan, K.D., Seals, D.R. Age-predicted maximal heart rate revisited. J Am Col Cardiol. 37: 153-56. 2001.

20. Clemente V y Gonzalez J: Four weeks of training with different aerobic workload distributions - Effect on aerobic performance. Eur. J. Sport Sci. 2014; 14(S1): S1-S7. DOI:10.1080/17461391.2011.635708.

21. De la Cruz B, López C, Naranjo J. Analysis of heart rate variability at rest and during aerobic exercise: a study in healthy people and cardiac patients. $\mathrm{Br}$ J Sports Med 2008; 42: 715-20.

22. Roberts AP, Y Cole JC. The effects of exercise and body armor on cognitive funtion in healthy volunteers. Mil Med. 178(5); 479-86. 2013.

23. Davis JM, Bailey SP. Possible mechanisms of central nervous system fatigue during exercise. Med Sci Sports Exerc. 1996; 29(1): 45-55.

24. Clemente V. Modificaciones de parámetros bioquímicos después de una maratón de montaña. Motricidad. Eur J Hum Mov 2011; 27: 75-83

25. Sjödin B, Jacobs I. Onset of blood lactate accumulation and marathon running performance. Int J Sports Med 1981; 2:23-6.

26. Hübner S. Combat shooting and self-defense. REDE Ed. Buenos Aires. 1984 40-112. 